\title{
Actividades para la enseñanza de las fuentes de energía en Educación Secundaria Obligatoria
}

\author{
Ma Jesús Arrebola Miranda, Ricardo Casas del Castillo y Francisco Javier Carrillo-Rosúa. \\ Universidad de Granada
}

Recepción: 12 de abril de 2015 | Revisión: 30 de mayo de 2015 | Aceptación/Publicación: 24 de julio de 2015 Correspondencia: arrebolamiranda@gmail.com | ricardocasas@ugr.es | fjcarril@ugr.es

Citar: Arrebola-Miranda, M.J., Casas, R. y Carrillo-Rosua, F.J. (2015) Actividades para la enseñanza de las fuentes de energía en Educación Secundaria Obligatoria. ReiDoCrea, 4(2), 148-153. http://hdl.handle.net/10481/37137

\begin{abstract}
Resumen: En este artículo se presenta un banco de actividades enfocado a la enseñanza de las fuentes de energía para $2^{\circ}$ curso de Educación Secundaria Obligatoria (ESO). Debido a la relevancia social que han adquirido en las últimas décadas las problemáticas medioambientales relacionadas con el uso de la energía y la escasez de recursos energéticos, se hace imprescindible formar una ciudadanía que se involucre activamente en la toma de decisiones bien fundamentadas respecto a un futuro que les afecta plenamente. Dado que la enseñanza tradicional se ha visto incapaz de alcanzar este objetivo, desde la Didáctica de las Ciencias Experimentales se aboga por la utilización de metodologías activas que favorecen la alfabetización científica. Las actividades planteadas están en consonancia con dichas metodologías, usando un enfoque Ciencia-Tecnología-Sociedad, partiendo de situaciones problemáticas reales, promoviendo el trabajo experimental con diseños del propio alumnado, enfatizando el aprendizaje colaborativo, etc. Pueden implementarse de forma aislada o formando secuencias de más de una actividad, permitiendo el desarrollo de competencias que implican la movilización de actitudes y comportamientos coherentes con un desarrollo sostenible de una sociedad cada vez más tecnificada y que se enfrenta a verdaderos y graves problemas económicos y ambientales.
\end{abstract}

Palabras clave: Didáctica de las Ciencias Experimentales | Educación Medioambiental

\section{ACTIVITIES FOR TEACHING ENERGY SOURCES IN SECONDARY EDUCATION}

\begin{abstract}
In this paper we present a bank of activities focused on the teaching of energy sources for Secondary Education (grade $8^{\text {th }}$ ). Due to the great relevance of environmental problems related with the use of energy and the scarcity of energy resources, it is essential to educate citizens on this topic. Citizens should involve in well-founded decision-making in respect to a future that affects themselves. Traditional teaching methods have revealed unable to achieve these goals. Thus, from Science Teaching, it is promoted the use of active methodologies that favor scientific and technological literacy. The proposed activities are in accordance with these active methodologies: using a ScienceTechnology-Society -STS- perspective, starting with real problematic situations, promoting the experimental work designed by the pupils themselves, emphasizing collaborative learning, etc. They can be implemented either isolated or as part of a sequence. They allow the development of competencies that build up attitudes and behaviors that are coherent with sustainability of societies, which are becoming increasingly technical and that face serious economic and environmental problems.
\end{abstract}

Keywords: Science Education | Environmental Education

\section{Introducción}

Se presenta una propuesta didáctica consistente en un "banco" de actividades diseñado para facilitar el proceso de enseñanza y aprendizaje de las fuentes de energía, dirigido a segundo curso de Educación Secundaria Obligatoria (ESO) en el marco de la Ley Orgánica de Educación (MEC, 2007), aunque puede ser adaptable a la Ley Orgánica para la Mejora de la Calidad Educativa (MECD, 2015).

Las sociedades humanas han ido aumentando sus demandas energéticas, estando ligadas las revoluciones industriales y tecnológicas a innovaciones en este campo. Las fuentes de energía, actuales y pasadas, se clasifican en renovables y no renovables. Estas últimas, además del agotamiento, plantean también otros problemas, como la contaminación que producen y el impacto que su uso puede causar al clima (combustibles fósiles), o su peligrosidad en caso de accidente y la gestión de sus residuos (nuclear de fisión). Son necesarias soluciones adecuadas y urgentes en la línea del uso racional de la energía y de la apuesta por las renovables. 
Hay que plantear, pues, soluciones a esta problemática, comenzando por cambios de actitudes que lleven a modificar nuestros comportamientos, intentando promover un desarrollo sostenible. Uno de los pilares fundamentales de todo ello es la educación.

En esta línea, se ha elaborado un "banco" de actividades que aborda la temática de las fuentes de energía, y que presenta asociados unos elementos curriculares que se inscriben en el marco del RD1631/2006 (MEC, 2007). Se ha buscado que el alumnado encuentre sentido a lo que está realizando, y que sea protagonista de su propio aprendizaje desarrollando competencias, siendo el docente un guía en este proceso.

\section{Marco teórico}

En el ámbito de las Ciencias de la Naturaleza existen numerosos trabajos en los que se analizan algunos aspectos relacionados con la enseñanza y aprendizaje de la energía en la ESO, pero son relativamente escasos los que se centran en las fuentes de energía, y más concretamente, en las renovables (García, Rodríguez, Solís Sánchez, y Ballenilla, 2007).

El proyecto "Energy Literacy" (U.S. Department of Energy, 2012) es una reciente iniciativa de alfabetización científica que pretende poner de manifiesto el rol que tiene la energía en el mundo y en nuestras vidas. Establece siete principios esenciales, amplias categorías de carácter científico, pero también social, que representan "grandes ideas". Cada uno de ellos está apoyado por un número variable de conceptos fundamentales (entre 6 y 8 ), constituyendo el marco conceptual de esta propuesta.

Aunque prácticamente todo el mundo está de acuerdo en que este es un tema que hay que tratar en la escuela, podemos plantearnos la misma cuestión que García et al. (2007): ¿Hay un adecuado tratamiento de la temática de la energía desde los primeros niveles? Por ejemplo, Rodríguez y García (2007) analizaron doce materiales didácticos con un total de 80 actividades para trabajar la energía. Estos autores concluyeron que la mayoría de ellas se referían a la descripción de fuentes de energía y de tipos de usos energéticos, así como a problemas asociados al consumo doméstico, mientras que el número de actividades que intentaban una aproximación más global y compleja al tema eran mucho menos numerosas.

Se presenta esta propuesta con el afán de superar esta deficiencia, especialmente por las estrategias metodológicas que articula. Así, se han diseñado actividades de distinta tipología que promueven el desarrollo de las competencias básicas (MEC, 2007). Las hay de carácter experimental, en las que el alumnado tendrá que realizar sus propios diseños, respondiendo también de esta manera a la conveniencia de la indagación en la enseñanza de las ciencias (Garritz, 2006). Otras responden a la filosofía CienciaTecnología-Sociedad (CTS), ya que, como señalan Domenech et al. (2003), la atención a las interacciones entre ciencia, tecnología y sociedad tienen que ser un aspecto esencial en la enseñanza de las fuentes de energía y el ahorro energético. Otras actividades encajarían en una metodología que responde al aprendizaje basado en problemas (ABP), entre cuyas ventajas encontramos que permiten adquirir habilidades de distinto rango cognitivo y facilitan la detección de ideas previas en el alumnado (Perales, 2010). Además, en todas las actividades se promueve el uso de las Tecnologías de la Información y la Comunicación (TIC), y se enfatiza la importancia del trabajo grupal del alumnado y la interacción entre iguales, de acuerdo con las tendencias actuales educativas y con la visión socioconstructivista de la enseñanza y aprendizaje de las ciencias (Badia y García, 2006). En la Tabla 1 se señala sinópticamente como las actividades propuestas incluyen las metodologías arriba mencionadas. 
Tabla 1. Relación entre las actividades y cada una de las metodologías empleadas.

\begin{tabular}{|c|c|c|c|c|c|c|}
\hline & Experimental & Indagación & CTS & ABP & $\begin{array}{l}\text { Uso de } \\
\text { las TIC }\end{array}$ & $\begin{array}{l}\text { Aprendizaje } \\
\text { colaborativo }\end{array}$ \\
\hline $\begin{array}{l}\text { 1. Construimos una } \\
\text { chimenea solar }\end{array}$ & $x$ & $x$ & & & $x$ & $x$ \\
\hline $\begin{array}{l}\text { 2. ¡Construye tu horno } \\
\text { solar! }\end{array}$ & $x$ & & & & $x$ & $x$ \\
\hline $\begin{array}{l}\text { 3. ¿Para qué utilizarías la } \\
\text { destilación solar? }\end{array}$ & $x$ & $x$ & $x$ & $x$ & $x$ & $x$ \\
\hline $\begin{array}{l}\text { 4. ¿Recurso energético es } \\
\text { igual a conflicto? }\end{array}$ & & $\mathbf{x}$ & $x$ & $x$ & $x$ & $x$ \\
\hline $\begin{array}{l}\text { 5. ¿Cuánta energía hay en } \\
\text { la comida de Marta? }\end{array}$ & & $x$ & $x$ & & $x$ & $x$ \\
\hline $\begin{array}{l}\text { 6. "Tu programa de } \\
\text { energía" }\end{array}$ & & $x$ & & & $x$ & $x$ \\
\hline
\end{tabular}

\section{Propuesta de actividades}

Se proponen seis actividades de duración y complejidad variables. En todas ellas se ha seguido la misma estructura (Arrebola, 2015):

- Título: Pensado para que intente reflejar lo que se va a realizar y que a la vez sea atractivo para el alumnado.

- Fundamentación: Trata de aportar la información científica que se trabajará, además de relacionar la actividad con las problemáticas sociales con las que está relacionada.

- Elementos curriculares: Tabla con los elementos curriculares (de acuerdo con el RD1631/2006 MEC, 2007) : competencias (con una breve justificación de cómo contribuye la actividad al desarrollo de las mismas), objetivos específicos (relacionados con los generales de la materia), contenidos y criterios de evaluación.

- Materiales y recursos: Los necesarios para realizar la actividad, partiendo del principio de procurar que fueran lo menos costosos posibles para favorecer su aplicabilidad.

- Descripción detallada de la actividad: De cara a que cualquier docente pueda implementarla de acuerdo con el diseño original, se describe pormenorizadamente la secuencia de acciones de la actividad y se propone una temporalización aproximada de la misma.

- Alternativas: En algunas actividades se incluye un apartado donde se proponen modificaciones o actuaciones que pueden completar $u$ ofrecer otra visión complementaria.

A continuación, se describen brevemente las actividades diseñadas:

Construimos una chimenea solar. Se comienza describiendo el funcionamiento de una chimenea solar, así como la importancia que ha tenido nuestro país en la invención y desarrollo de este tipo de estructuras. Después de fomentar una lluvia de ideas a partir de preguntas formuladas en torno a un modelo a escala que el docente llevará a clase, se presentará una animación flash (Eroski Consumer, 2011) donde se muestra un proyecto de construcción de una torre solar en Fuente el Fresno (Ciudad Real). A continuación el alumnado debe construir su propio modelo de chimenea solar, siguiendo las indicaciones aportadas por el docente en un vídeo de Youtube (https://www.youtube.com/watch?v=QBVg9iQ04ns). Con ella, se propone que el alumnado mida la temperatura del interior cuando está en funcionamiento, y genere una gráfica que muestre cómo varía en función del tiempo. Para terminar, se les pide 
que, utilizando TIC, busquen información sobre la historia de la torre solar, y en un breve informe comenten en qué consiste el proyecto Enviromission (Enviromission Limited, 2015).

Como alternativa, se puede utilizar el modelo de chimenea solar construido para analizar importantes fenómenos naturales que se estudian en la ESO, como la propagación del calor por convección, que explica buena parte de la dinámica de la atmósfera.

¡Construye tu horno solar! Después de una pequeña introducción, se proponen una serie de preguntas acerca del funcionamiento del horno solar, relacionándolo con el efecto invernadero. Estas no se responden en ese momento, aunque generarán un debate entre el alumnado. Posteriormente se le guiará en la construcción de su propio horno. Una vez fabricado, podrán probar su funcionamiento colocando una olla llena de agua. En el patio del centro educativo deberán medir la temperatura del interior y la ambiente cada cinco minutos durante una media hora, anotando los resultados en una tabla y comentándolos en clase. Posteriormente se elaborará un informe que incluya una gráfica con los datos tomados, y donde se describan los procesos que intervienen, las conclusiones y las posibles aplicaciones así como las ventajas y desventajas. Para completar el informe tendrán que buscar información sobre el horno solar, su funcionamiento y ventajas e inconvenientes usando diferentes fuentes, incluidas las TIC.

¿Para qué utilizarías la destilación solar? Esta actividad sigue la filosofía CTS, y está pensada para implementarse junto con la siguiente actividad, por lo que es recomendable, si se va a realizar la número 4, incluir previamente esta. En primer lugar se presentará, un modelo de destilador solar elaborado por el docente, y se generará a continuación una lluvia de ideas sobre sus posibles utilidades en contextos reales. Posteriormente se propondrá al alumnado que lo utilice para depurar el agua de dos recipientes (una salada y otra turbia). Después de explicar entre todos lo que ha ocurrido y el fundamento científico de lo observado, tendrán que buscar información en forma de datos o gráficas sobre la cantidad de agua en distintos reservorios que hay en el planeta, y qué porcentaje está disponible para el ser humano. Por último se les plantea una pregunta: ¿cómo este experimento podría ayudar al problema de la falta de agua potable en el mundo? Se añade la posibilidad de visualizar el documental "Por un trago de agua" (https://www.youtube.com/watch?v=9dIVSYxtoZ8) que muestra la grave situación que se está viviendo en numerosos lugares del planeta debido a la escasez de agua. Posteriormente, el alumnado puede contestar a una serie de preguntas sobre este tema de cara a relacionar y a integrar los aspectos científicos y sociales de la actividad.

¿Recurso energético es igual a conflicto? Se plantea la relación existente entre numerosos recursos (materiales o energéticos) y conflictos existentes actualmente a nivel mundial. Se les mostrará alguna noticia relacionada con la escasez de agua (https://www.youtube.com/watch?v=LC8y8i2P1y0) e información sobre la distribución de agua potable en el mundo (Conflictos por recursos, 2013). Usando las TIC, se les propondrá que busquen información sobre los conflictos relacionados con la falta de agua potable, y a continuación se les presentará alguna noticia sobre situaciones reales relacionadas con su escasez. Después de discutir sobre ella, tendrán que presentar un proyecto en forma de maqueta en el que propongan alguna solución.

¿Cuánta energía hay detrás de la comida de Marta? En esta actividad se pretende que el alumnado sea consciente del gasto energético que se lleva a cabo en un día cualquiera de sus vidas mediante una estimación del mismo. Se comienza con una 
lluvia de ideas para que indiquen qué "tipos" de energía están utilizando en ese mismo momento. Se presenta un personaje ficticio, "Marta" (que puede ser cualquiera de ellos), que se prepara tres comidas al día. Con los datos de la potencia de los electrodomésticos que tiene que utilizar, han de estimar la energía empleada, y a partir de ella, la emisión equivalente de $\mathrm{CO}_{2}$. A partir de esta tarea, se les pedirá que, por grupos, realicen un listado de las actividades que llevan a cabo a lo largo de un día, y estimen cuánta energía han consumido, relacionándola además con las distintas fuentes de donde proceden. Además, han de buscar información sobre la cantidad de energía consumida en España, cuáles son las principales fuentes, etc. Todo ello ha de quedar recogido en un informe con sus correspondientes tablas y gráficas, empleando software adecuado. Siguiendo la perspectiva CTS, posteriormente se les presentará dos imágenes, una de "Marta" cocinando, y otra de una niña de Mozambique preparando la comida para su familia. Se les planteará una serie de preguntas para generar un debate que les debe llevar a darse cuenta del despilfarro energético de nuestra vida cotidiana en el mundo occidental. Por último, se les pedirá que elaboren un mural con acciones que pueden realizar a lo largo del día para ahorrar energía.

Tu programa de energía. Consiste en un la realización de un podcast por parte del alumnado sobre las fuentes de energía y el ahorro energético. Para ello han de informarse sobre las diferentes fuentes de energía, su definición, producción, transporte y distribución, así como sus usos, ventajas e inconvenientes. Toda esta información se incluirá en el "programa radiofónico" que constituye el podcast. Lo harán mediante entrevistas simuladas, utilizando como metodología el juego de rol, ya que un alumno será el entrevistador y otro el entrevistado. Finalmente, entre toda la clase se creará una lista con acciones diarias que pueden realizar para contribuir al ahorro energético. Se creará un podcast entre todos, y posteriormente cada uno se decidirá por una acción, de entre las propuestas.

\section{Referencias}

Arrebola Miranda, M. J. (2015). La enseñanza de las fuentes de energía renovables en Educación Secundaria. Granada: Universidad de Granada. http://digibug.ugr.es/handle/10481/36588

Conflictos por recursos. (s.f.). Recuperado el 26-06-2015 de: http://www.conflictosporrecursos.es/. (Archived by WebCite® at http://www.webcitation.org/6ZZppUZzJ).

Badia, A., y García, C. (2006). Incorporación de las TIC en la enseñanza y el aprendizaje basados en la elaboración colaborativa de proyectos. Revista de Universidad y Sociedad del Conocimiento, 3(2), 42-54.

Doménech, J. L., Gil-Pérez, D., Gras, A., Guisasola, J., Martínez-Torregrosa, J., Salinas, J., Trumper, R. y Valdés, P. (2003). Enseñanza de la energía: una propuesta de debate para un replanteamiento global. Caderno Brasileiro de Ensino de Física, 20(3), 285-311.

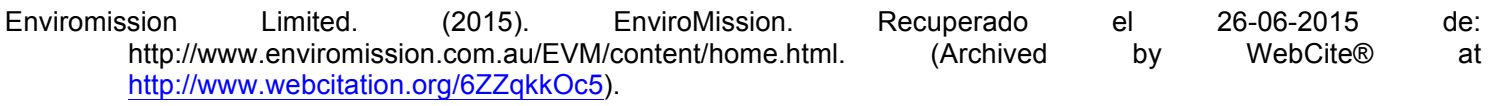

Eroski Consumer. (2011). Infografía: Torre solar. Recuperado el 26-06-2015 de: http://www.consumer.es/web/es/medio_ambiente/energia_y_ciencia/2009/04/12/184638.php. (Archived by WebCite® at http://www.webcitation.org/6ZZf5NqNG).

García Díaz, J. E., Rodríguez, F., Solís Sánchez, M. C., y Ballenilla García, F. (2007). Investigando el problema del uso de la energía. Investigación en la Escuela, 63, 29-45.

Garritz, A. (2006). Naturaleza de la ciencia e indagación: cuestiones fundamentales para la educación científica del ciudadano. Revista Iberoamericana de Educación, 42, 127-152.

Ministerio de Educación y Ciencia (2007). Real Decreto 1631/2006, de 29 de diciembre, por el que se establecen las enseñanzas mínimas de la Educación Secundaria Obligatoria. Boletín Oficial del Estado (B.O.E.), de 5 de enero de $2007, n^{\circ} 5,677-773$. 
Ministerio de Educación, Cultura y Deporte (2015). Real Decreto 1105/2014, de 26 de diciembre, por el que se establece el currículo básico de la Educación Secundaria Obligatoria y del Bachillerato. Boletín Oficial del Estado (B.O.E.), de 3 de enero de 2015, n³, 169 - 546.

Perales Palacios, F. J. (2010). La resolución de problemas en la didáctica de las ciencias experimentales. Revista educación y pedagogía, 10(21), 120-143.

Rodríguez, F., y García, J. E. (2007). ¿Qué dificultades encuentran los docentes para trabajar la energía en el ámbito de la educación ambiental formal? En II Congreso Internacional de Educación, Energía y Desarrollo Sostenible. As Pontes de García Rodríguez 27, 28 y 29 de junio de 2007.

U.S. Department of Energy. (2012). Energy Literacy: Essential Principles and Fundamental Concepts for Energy Education. Retrieved from http://energy.gov/eere/education/energy-literacy-essential-principles-andfundamental-concepts-energy-education. (Archived by WebCite ${ }^{\circledR}$ at http://www.webcitation.org/6alGLTQId).

Vilches, A., y Gil-Pérez, D. (2010). ¿Cómo puede contribuir la educación a la construcción de un futuro sostenible? Revista Eureka sobre enseñanza y divulgación de las ciencias, 7( ${ }^{\circ}$ extraordinario), 297-315. 